\title{
Upper limit on the transition temperature for non-ideal Bose gases
}

\author{
Wu-Sheng Dai ${ }^{1,2}$ *and Mi Xie ${ }^{1,2} \dagger$ \\ ${ }^{1}$ Department of Physics, Tianjin University, Tianjin 300072, P. R. China \\ ${ }^{2}$ LiuHui Center for Applied Mathematics, Nankai University \& Tianjin University, Tianjin 300072, P. R. China
}

\begin{abstract}
In this paper we show that for a non-ideal Bose gas there exists an upper limit on the transition temperature above which Bose-Einstein condensation cannot occur regardless of the pressure applied. Such upper limits for some realistic Bose gases are estimated. This result implies that there may also exist an upper limit on the transition temperature of superconductors.
\end{abstract}

*Email: daiwusheng@tju.edu.cn

${ }^{\dagger}$ Email: xiemi@tju.edu.cn 
Bose-Einstein condensation is the first purely statistically derived example of a phase transition. The problem of Bose-Einstein condensation in ideal and non-ideal Bose gases has interested physicists both from practical viewpoint and academic viewpoint for many years [1, 2]. Especially, in recent years Bose-Einstein condensation in the dilute repulsive Bose gas has attracted wide attention [3]. In this paper, we will show that for a non-ideal Bose gas there exists an upper limit on the transition temperature, above which the condensation cannot occur regardless of the pressure applied, though there is no upper limit on the transition temperature for an ideal Bose gas. For an ideal Bose gas the condensation can occur in any temperature so long as the pressure is large enough. In this paper, we will place an upper limit on the transition temperature for Bose-Einstein condensation. We also estimate such upper limits for some realistic Bose gases which are of course non-ideal gases.

The reason why transitions may occur in Bose gases is that there exist exchange interactions among the particles, and the effect of exchange interactions is attractive; in other words, a Bose gas will exhibit attractive forces, a pure quantum effect, between its gas molecules. The competition between the exchange interaction and the thermal motion of molecules determines the state of the system: when the exchange interaction dominates, Bose-Einstein condensation takes place. The strength of the exchange interaction, roughly speaking, is determined by the overlap among the wavefunctions of the gas molecules. The overlap of the wavefunctions can be roughly described by the magnitudes of the mean space between particles and the mean thermal wavelength which measures the mean spatial extent of the wavefunction of a particle. In the case of the ideal gas, there are no interparticle interactions, so the separation between particles can take on any small value. Therefore, the wavefunctions can overlap to any extent by reducing the distance between particles no matter how high the temperature, or no matter how short the wavelength, may be. As a result, ideal Bose gases can display Bose-Einstein condensation at any temperature so long as the density is high enough. That is to say, for ideal Bose gases there is no upper limit on the transition temperature.

For non-ideal Bose gases, besides exchange interactions, there also exist interparticle interactions. The interparticle interaction in a realistic gas is attractive when the molecules are moderately far apart, and is repulsive when they are close together. Especially, the repulsion will become so strong that it can be idealized as infinite when the interparticle spacing is close to a certain value. Consequently, there exists a lower limit on the interparticle spacing due to the interparticle interaction, and thus the overlap of the wavefunctions is limited. There must exist such a temperature above which the corresponding mean thermal wavelength will be always shorter than the interparticle spacing, and, then, the wavefunctions of the molecules do not overlap. That is to say, for a non-ideal Bose gas, in contrast to the ideal case, there is an upper limit on the transition tem- 
perature above which Bose-Einstein condensation cannot occur regardless of the pressure applied. It should be emphasized that, all realistic gases must have interparticle interactions among their molecules, so for any realistic Bose gas there exists an upper limit on the transition temperature.

The approach for obtaining the upper limit on the transition temperature for a non-ideal Bose gas is based on the analogy between Bose-Einstein condensation and the gas-liquid transition [4. There exists an upper limit on the transition temperature for gas-liquid transition, called critical temperature (the word "critical temperature" here means the upper limit on the transition temperature, not the phase transition temperature, though the transition temperature is also called "critical temperature"). The existence of the upper limit on the transition temperature in the gasliquid transition is a result of the existence of the interparticle interaction. The interparticle interaction is attractive at a certain distance, which causes the gas to condense into a liquid. Such an interaction, however, will become repulsive at a very small distance, and consequently the potential energy has a minimum value. Therefore, when the temperature is higher than a certain value - the upper limit on the transition temperature - the gas cannot be liquefied regardless of the pressure applied.

One approach for calculating the upper limit on the transition temperature for the gas-liquid transition is first to obtain the van der Waals equation by calculating the virial expansion of the equation of state for the non-ideal gas, and then to calculate such an upper limit on the transition temperature based on the van der Waals equation [5]. In principle, the result obtained in such a way is valid only for the gas phase and cannot be used to describe the gas-liquid transition since in gases the interaction between particles is weak but in liquids the interaction between particles is strong. However, after a modification which removes the unphysical part of the van der Waals equation, which implies a negative compressibility, one can find a result which can be used to describe the transition between the liquid and gaseous states. Especially this result can be used to discuss the upper limit on the transition temperature [6].

In this paper, we use the hard-sphere Bose gas as a simplified model for non-ideal Bose gases. This is because the essential reason for the existence of an upper limit on the transition temperature is the existence of the repulsive core in the interparticle interaction. The result obtained by the hard-sphere model is also valid for more general interparticle interactions since a particle that is spread out in space sees only an averaged effect of the potential, and, thus often a complete knowledge of the detailed interaction potential is not necessary for a satisfactory description [7]. Or, from the viewpoint of quantum mechanics, due to the low collision energy of collisions between molecules in a Bose gas near the transition temperature, one can use the $s$-wave approximation which is shape-independent. The hard-sphere gas, as a simplified model, is of great value for investigating the more general theory of non-ideal gases, especially after Lee and Yang pioneered 
the study of interacting bosons [8]; it is often used as an efficient tool for studying Bose-Einstein condensation, both for dilute atomic gases and liquid helium [9].

In the following we estimate the upper limit on the transition temperature for the hard-sphere Bose gas. The equation of state for hard-sphere gases is [10]

$$
\begin{aligned}
\frac{P}{k T} & =\frac{1}{\lambda^{3}} g_{5 / 2}(z)-\frac{2 a}{\lambda^{4}} g_{3 / 2}^{2}(z), \\
\frac{N}{V} & =\frac{1}{\lambda^{3}} g_{3 / 2}(z)-\frac{4 a}{\lambda^{4}} g_{3 / 2}(z) g_{1 / 2}(z),
\end{aligned}
$$

where $a$ is the scattering length, $\lambda=h / \sqrt{2 \pi m k T}$ is the mean thermal wavelength, and $g_{\sigma}(z)$ is the Bose-Einstein integral. For obtaining the upper limit on the transition temperature for the hard-sphere Bose gas, we need to find the virial expansion of Eq. (11). The second virial coefficient is 6 ]

$$
b_{2}=\frac{1}{4 \sqrt{2}}-2 \frac{a}{\lambda} .
$$

Then the equation of state approximately reads

$$
\frac{P V}{N k T}=1-\left(\frac{1}{4 \sqrt{2}}-2 \frac{a}{\lambda}\right) \frac{N}{V} \lambda^{3} .
$$

By Eq. (2) we can draw the $P-V$ diagram (Figure 1). Corresponding to $\lambda>8 \sqrt{2} a$ and $\lambda<8 \sqrt{2} a$ there are two types of isotherms: one type of the isotherms is monotonically decreasing, and the other type of isotherms has a turning point - a maximum point - on the curve. The isotherms that are not monotonic correspond to the transition. Evidently, there is an unphysical part, corresponding to $d P / d V>0$, on each of such isotherms. The correct result of this part should be isobaric. Between this two type of isotherms, there is a critical isotherm which corresponds to the upper limit on the transition temperature, $T_{u}$. Such an upper limit can be determined by the relation $\lambda=8 \sqrt{2} a$ :

$$
T_{u}=\frac{h^{2}}{256 \pi m k} \frac{1}{a^{2}} .
$$

When $T<T_{u}$, so long as the pressure is large enough, the transition may occur. However, when $T>T_{u}$ (in this case the pressure $P$ is a monotonically decreasing function of the volume $V$ ), the transition will not occur regardless of how large may be the pressure. It should be emphasized that in the case of the gas-liquid transition, instead of the "peak" which corresponds to the transition there is a "kink" in the isotherm. This is because when Bose-Einstein condensation occurs, the system is in the phase which is a mixture of two phases all along the whole process of the transition till the temperature tends to the absolute zero; in other words, in contrast to the gas-liquid transition, there is no unmixed condensed phase in a Bose gas due to the unachievable absolute zero. 


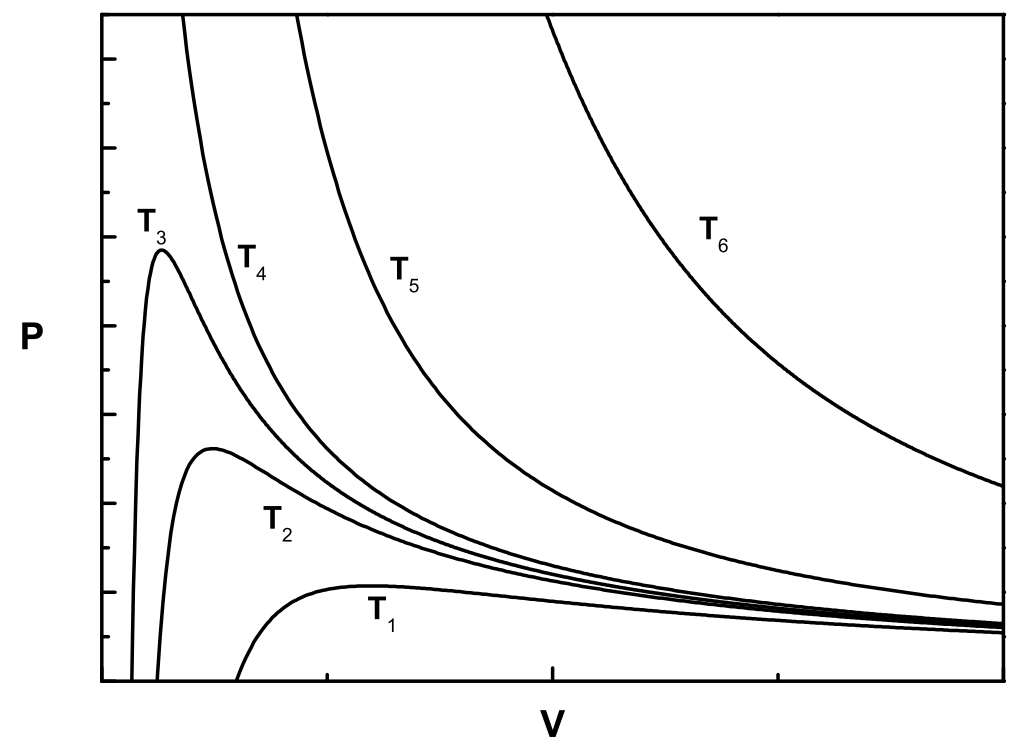

Figure 1. $P-V$ diagram of Eq. (2). $T_{1}<T_{2}<T_{3}<T_{u}$ and $T_{6}>T_{5}>T_{4}>T_{u}$.

The expression for the upper limit on the transition temperature, Eq. (3), shows that $T_{u}$ is inversely proportional to the square of the scattering length, $a^{2}$. This implies that the stronger the interaction, the lower the upper limit on the transition temperature. Especially, for ideal gases, there are no interparticle interactions and so the scattering length $a=0$; in this case $T_{u} \rightarrow \infty$, i.e., there is no restriction on the transition temperature in ideal Bose gases. Such a conclusion agrees with the above qualitative arguments.

Based on the above result we can estimate the upper limits on the transition temperatures for some realistic Bose gases, ${ }^{1} \mathrm{H},{ }^{23} \mathrm{Na}$, and ${ }^{87} \mathrm{Rb}$; Bose-Einstein condensation in such gases have been observed [11. The spin-triplet scattering lengths of the atoms ${ }^{1} \mathrm{H},{ }^{23} \mathrm{Na}$, and ${ }^{87} \mathrm{Rb}$ are $a^{H}=0.065 n m, a^{N a}=2.8 n m$, and $a^{R b}=5.4 n m$, respectively [12. By Eq. (3), we have

$$
\begin{aligned}
T_{u}^{H} & =5.6 K, \\
T_{u}^{N a} & =0.13 m K, \\
T_{u}^{R b} & =9.3 \mu K .
\end{aligned}
$$

Comparing these results to the transition temperatures of such atomic gases, $T_{c}^{H}=50 \mu K, T_{c}^{N a}=$ $2 \mu k$, and $T_{c}^{R b}=0.67 \mu K\left[12\right.$, we can see that the various $T_{u}$ obtained above are higher than the transition temperatures. This agrees with the conclusion drawn above: $T_{u}$ is the upper limit on 
$T_{c}$.

In conclusion, in this paper we show a difference between ideal and non-ideal Bose gases: a nonideal Bose gas has an upper limit on the transition temperature, but an ideal one has none. If the temperature is higher than such an upper limit, a non-ideal Bose gas cannot display Bose-Einstein condensation. We estimate the upper limit on transition temperatures for some realistic Bose gases and compare them to the transition temperatures measured in some recent experiments.

Furthemore, the above result also implies that there may also exist an upper limit on the transition temperature of superconductors, if superconduction can be viewed as a kind of BoseEinstein condensation.

Acknowledgement We are very indebted to Dr G. Zeitrauman for his encouragement. This work is supported in part by NSF of China, under Project No.10605013 and No.10675088.

\section{References}

[1] A. Griffin, D. W. Snoke, and S. Stringari, Bose-Einstein Condensation, Cambridge University Press, Cambridge, U.K., 1995.

[2] R.M. Ziff, G.E. Uhlenbeck, and M. Kac, Phys. Rep. C 32 (1977) 169-248; F. Dalfovo, S. Giorgini, L.P. Pitaevskii and S. Stringari, Rev. Mod. Phys. 71 (1999) 463-512; A.J. Leggett, Rev. Mod. Phys. 73 (2001) 307-356; V.A. Zagrebnov, J.-B. Bru, Phys. Rep. 350 (2001) 291434; V. Bagnato and D. Kleppner, Phys. Rev. A 44 (1991) 7439-7441; V. Bagnato, D.E. Pritchard, and D. Kleppner, Phys. Rev. A 35 (1987) 4354-4358.

[3] M. Holzmann, J.-N. Fuchs, G.A. Baym, J.-P. Blaizot, F. Laloë, C. R. Physique 5 (2004) 21-37; G. Baym, J.-P. Blaizot, M. Holzmann, F. Laloë, and D. Vautherin, Eur. Phys. J. B 24 (2001) 107-124; S. Ledowski, N. Hasselmann, and P. Kopietz, Phys. Rev. A 69 (2004) 061601(R)-1-4; P. Arnold, G. Moore, and B. Tomázik, Phys. Rev. A 65 (2002) 013606-1-18; J.O. Andersen, Rev. Mod. Phys. 76 (2004) 599-639.

[4] B. Kahn and G.E. Uhlenbeck, Physica 5 (1938) 399-416; F. Mohling, Statistical Mechanics: Methods and Applications, John Wiley and Sons, New York, 1982.

[5] W. Greiner, L. Neise, and H Stöcker, Thermodynamics and Statistical Mechanics, SpringerVerlag, New York, 1994.

[6] R.K. Pathria, Statistical Mechanics, 2nd Ed. Butterworth-Heinemann, Oxford, 1996. 
[7] K. Huang and C.N. Yang, Phys. Rev. 105 (1957) 767-775; K. Huang, C.N. Yang and J.M. Luttinger, Phys. Rev. 105 (1957) 776-784.

[8] T.D. Lee and C.N. Yang, Phys. Rev. 112 (1958) 1419-1429.

[9] W. Krauth, Phys. Rev. Lett. 77 (1996) 3695-3699; G. Baym, J.-P. Blaizot, M. Holzmann, F. Laloë, and D. Vautherin, Phys. Rev. Lett. 83 (1999) 1703-1706.

[10] T.D. Lee and C.N. Yang, Phys. Rev. 116 (1959) 25-31; T.D. Lee and C.N. Yang, Phys. Rev. 105 (1957) 1119-1120.

[11] M.H. Anderson, J.R. Ensher, M.R. Matthews, C.E. Wieman, and E.A. Cornell, Science 269 (1995) 198-201; C.C. Bradley, C.A. Sackett, J.J. Tollett, and R.G. Hulet, Phys. Rev. Lett. 75 (1995) 1687-1690; K.B. Davis, M.-O. Mewes, M.R. Andrews, N.J. van Druten, D.S. Durfee, D.M. Kurn, and W. Ketterle, Phys. Rev. Lett. 75 (1995) 3969-3973; D.G. Fried, T.C. Killian, L. Willmann, D. Landhuis, S.C. Moss, D. Kleppner, and T.J. Greytak, Phys. Rev. Lett. 81 (1998) 3811-3814; J.R. Anglin, W. Ketterle, Nature 416 (2002) 211-218.

[12] D. Kleppner, T.J. Greytak, T.C. Killian, D.G. Fried, L. Willmann, D. Landhuis, and S.C. Moss, in Proceedings of the International School of Physics "Enrico Fermi," Course CXL edited by M. Inguscio, S. Stringari, and C.Wieman, IOS Press, Amsterdam, 1999. 